Urologe 2011 · 50:1247

DOI 10.1007/s00120-011-2615-6

Online publiziert: 11. September 2011

(c) Springer-Verlag 2011

G. Lümmen

Klinik für Urologie und Kinderurologie, St. Josef-Hospital Troisdorf, Troisdorf

\title{
Prävention - ein zentrales Anliegen der Urologen
}

Eine weitere sog. „Volkskrankheit“ stellt das benigne prostatische Syndrom (BPS) dar. Dieses Syndrom entsteht durch die benigne Prostatahyperplasie und führt zu fast 6 Mio. Konsultationen/Jahr allein in Deutschland. Der Artikel von Oelke und Madersbacher vertieft sehr interessante Ansätze zur primären und sekundären Prävention des BPS.

Auch das Harnsteinleiden ist ein nicht zu unterschätzender gesundheitsökonomischer Faktor. Aus diesem Grund werden die spezifischen Maßnahmen zur Metaphylaxe bei den verschiedenen Steinerkrankungen aufgeführt. Anhand eines Berechnungsmodells wird der volkswirtschaftliche Nutzen einer solchen Sekundärprävention von Harnsteinen dargelegt.

Die erektile Dysfunktion ist eine Erkrankung mit hoher Prävalenz, beginnend mit ca. 10\% der 55-jährigen Männer bis hin zu 75\% bei 8o-jährigen Männern und ist somit von zentraler Bedeutung. Gerade die Maßnahmen zur Primär- und Sekundärprävention der erektilen Dysfunktion, insbesondere in Bezug auf die kardiovaskuläre Pathophysiologie der Erkrankung, zeigen anschaulich den ganzheitlichen Ansatz der Urologinnen und Urologen, entgegen der sog. „Reparaturmedizin“.

„Last but not least“ können auch uroonkologische Erkrankungen sowohl im Sinne einer primären als auch einer sekundären Prävention verringert oder sogar vermieden werden. Die Arbeitsgruppe aus dem Arbeitskreis Prävention, Umwelt und Komplimentärmedizin zeigt am Beispiel des Prostatakarzinoms, dass weniger die Einnahme von bestimmten Spurenelementen oder Vitaminen sinnvoll ist, als vielmehr die Änderung des sog. „Life- styles“. Darunter versteht man eine maßvolle ballaststoffreiche Ernährung, $\mathrm{Ge}$ wichtsreduktion, körperliche Aktivität, Nikotinverzicht und mäßiger Alkoholkonsum. Interessanterweise sind diese Änderungen der Lebensgewohnheiten in allen Beiträgen zur Prävention, mit Ausnahme der Prävention von HWI, in gleicher Weise als präventiv aufgeführt.

Die vorgestellten Beiträge sollen Sie in der täglichen Diskussion mit den Patienten und Kollegen unterstützen und Ihren ganzheitlichen Ansatz in der Medizin als Urologinnen und Urologen unterstreichen.

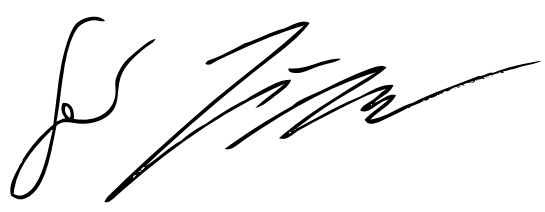

G. Lümmen

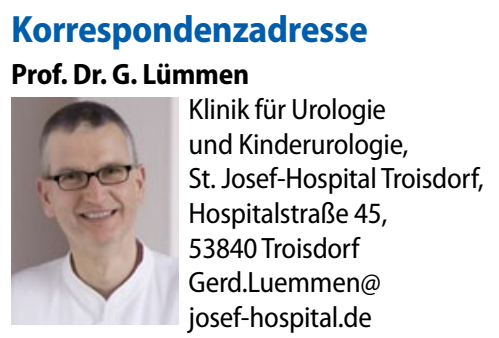

